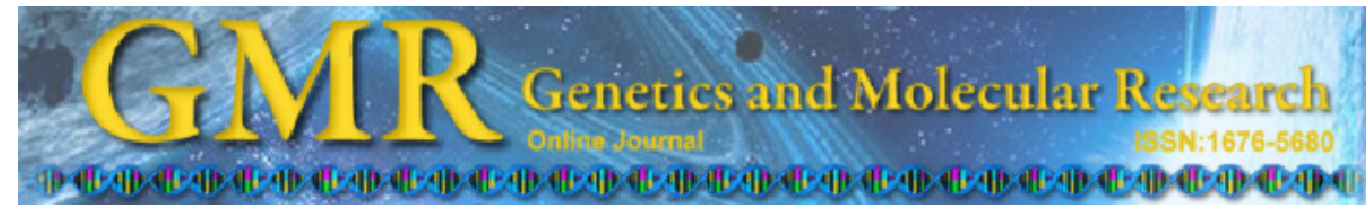

\title{
Frequency of $M D R 1$ single nucleotide polymorphisms in a Jordanian population, including a novel variant
}

O.F. Khabour ${ }^{1}$, K.H. Alzoubi ${ }^{2}$, S.I. Al-Azzam ${ }^{2}$ and N.M. Mhaidat ${ }^{2}$

${ }^{1}$ Department of Medical Laboratory Sciences, Faculty of Applied Medical Sciences, Jordan University of Science and Technology, Irbid, Jordan ${ }^{2}$ Department of Clinical Pharmacy, Faculty of Pharmacy, Jordan University of Science and Technology, Irbid, Jordan

Corresponding author: O.F. Khabour

E-mail: khabour@just.edu.jo

Genet. Mol. Res. 12 (1): 801-808 (2013)

Received July 2, 2012

Accepted November 23, 2012

Published March 13, 2013

DOI http://dx.doi.org/10.4238/2013.March.13.9

\begin{abstract}
The multidrug resistance gene (MDR1 or $A B C B 1)$ codes for P-glycoprotein, which plays an important role in regulating absorption, distribution, and elimination of drugs. We examined MDRI gene variants in 100 unrelated subjects from various regions of Jordan. The $M D R 1$ gene was scanned using direct sequencing. Six rare variants in $M D R 1$ were detected, including a new variant, T3075A. This variant did not affect the protein sequence (synonym for threonine). Among the common SNPs, the frequencies of rs 1128503 (C1236T) genotypes were: 0.23 (CC), 0.41 (CT) and 0.36 (TT). For the rs2032582 (G2677T) SNP, genotype frequencies were 0.38 for GG, 0.45 for GT, 0.13 for TT, 0.03 for GA, and 0.01 for TA, whereas for rs 1045642 (C3435T), genotype frequencies were 0.17 for $\mathrm{CC}, 0.5$ for $\mathrm{CT}$ and 0.33 for TT. The observed distribution of the common variants in the Jordanian population was within the range detected in other populations. These data on MDRl gene variants in the Jordanian population will be useful for investigations on response to P-glycoprotein substrate drugs.
\end{abstract}

Key words: MDR1; SNP; Jordan; Allele; Gene 


\section{INTRODUCTION}

Pharmacogenetics - the study of genetic variation and its effects on drug response - is expected to play a key role in individualizing patient care through "personalized medicine" (Roses, 2008). Pharmacogenetic investigations usually focus on drug-metabolism genes involved in specific disease. The multi-drug resistance gene (MDR1 or $A B C B 1$ ) spans 28 exons on chromosome 7; its cDNA consists of 3843 bp (Fojo et al., 1986). MDR1 encodes P-glycoprotein $(\mathrm{P}-\mathrm{gp}$ ), which is a large, $170-\mathrm{kD}$ transmembrane protein composed of 2 homologous halves, each of which contains 6 transmembrane domains and an intercellular binding site for ATP; thus, it functions as an ATP-dependent efflux pump (Higgins et al., 1997). It was first detected in cancer cells where it is responsible for multiple resistance to anticancer agents (He et al., 2011); however, it is also highly expressed in normal tissues such as the brain, liver, kidney, lymphocytes, placenta, gut, and testes (Cascorbi, 2011). P-gp plays an important role in regulating drug absorption, distribution, and elimination. It mediates the energydependent efflux of xenobiotics in epithelial tissues throughout the human body including the intestinal mucosa, liver canalicular membrane, and kidney proximal tubules, as well as blood-tissue barriers such as the brain and placenta (He et al., 2011). P-gp efflux, therefore, may decrease intestinal absorption, increase biliary excretion and renal tubular secretion, and impair distribution of various drugs to the brain.

P-gp exhibits wide substrate specificity for structurally different drugs and thus mediates resistance to a variety of drugs including anti-arrhythmics, antifungals, calcium channel blockers, chemotherapeutic agents, hormones, immunosuppressants, and HIV-protease inhibitors (Kuypers et al., 2008). Because P-gp is found in tissues important for drug disposition, variations in expression and function of P-gp due to MDR1 polymorphisms may influence drug pharmacokinetics and therapeutic efficacy.

MDRl gene expression is highly variable between subjects from the same as well as different races, and many variants have been identified (Hattori et al., 2007; Sipeky et al., 2011). For example, among Caucasians, the C3435T polymorphism in exon 26 correlates with expression of $\mathrm{P}$-gp in the intestine, and people who are homozygous for the $\mathrm{T}$ allele show more than 2-fold lower duodenal P-gp protein expression levels compared with $\mathrm{C}$ homozygotes. Since concentrations of P-gp in the intestine determine the extent of drug absorption, genotype-related differences in bioavailability are seen for several drugs (e.g., digoxin) (Chen et al., 2011; Li et al., 2011; Yan et al., 2011; Ponnala et al., 2012). Substantial differences in allele frequencies have been reported in different racial groups. For example, significantly higher frequencies of the C/C genotype of C3435T SNP were reported in West Africans and African Americans than in White Americans (Chelule et al., 2003; Lewis et al., 2007). In this study, we scanned the MDRl gene to identify variants in the Jordanian population. In addition, the distribution of common variants was compared to that reported in other populations.

\section{MATERIAL AND METHODS}

\section{Subjects}

Unrelated subjects of both sexes $(\mathrm{N}=100)$ were recruited randomly from different 
regions of Jordan. Random selection was carried out based on geographical distribution and population density. All participants were asked to provide written informed consent. The study was approved by the Institutional Review Board (IRB) of the Jordan University of Science and Technology.

\section{DNA extraction}

Blood samples $(5 \mathrm{~mL})$ were collected in EDTA tubes for DNA extraction. Genomic DNA was extracted with the Wizard DNA Extraction Kit (Promega, Madison, USA) according to the manufacturer protocol. DNA concentrations were measured using a SmartSpect ${ }^{\mathrm{TM}}$ 3000 (Bio-Rad, Hertfordshire, UK) and samples were stored at $-20^{\circ} \mathrm{C}$ prior to use.

\section{PCR amplification of $M D R 1$ axons}

Amplification of $M D R 1$ exons was carried out as previously described (Kim et al., 2001) with the following modifications: for exon 8 , the following primers were used: F: 5'-TAGCGTATGCAAAAGCTGGA-3' and R: 5'-TCTGAAGGGCATTTGAGAAGA-3', and for exon 18, F: 5'-CCAGGATGGGTTCTTCACTG-3' and R: 5'-CCCCCAGTTGAATAATGA TG-3'. In each reaction, approximately $100 \mathrm{ng}$ genomic DNA, $1 \mu \mathrm{M}$ of each forward and reverse primer, and green PCR master mix (Promega) were used in a final reaction volume of 25 $\mu \mathrm{L}$. PCR products were detected after electrophoresis on $2 \%$ agarose.

\section{Sequencing of MDR1 allelic variants}

Purification of PCR products was carried out using the PCRquick-Spin ${ }^{\mathrm{TM}}$ PCR Product Purification system as described by the manufacturer (INTro Biotechnolgy, Korea).

The purified PCR products were fully sequenced using the Big Dye Terminator Cycle Sequencing Kit version 3.1 (QIAquick, Germany). The ABI 3700 DNA Analyzer (Applied Biosystems) was used for sequencing. DNA sequences were compared to the reference sequence (NM_000927.4) stored in the Ensembl Genome Browser (http://www.

ensembl.org/index.html). Presence of a gene variant was confirmed by sequencing of the reverse strand. Sequencing results were analyzed using the ChromasPro version 1.34 software (http://www.technelysium.com.au/ChromasPro.html). Finally, the effect of the identified novel variant was analyzed with the STARORF software (Massachusetts Institute of Technology, UK).

\section{Statistical analysis}

Genotype distributions were analyzed for Hardy-Weinberg equilibrium. The SPSS 15.0 statistical software package (SPSS Inc., Chicago, IL, USA) was used for statistical analysis. P values smaller than 0.05 were considered to be significant.

\section{RESULTS}

Jordan is a small country in Southwest Asia and is classified as a low-income country. 
The population is predominantly Arab (98\%), mostly urban (70\%) (Khabour et al., 2009). As of the 2010 census, the total population of Jordan was 6.13 million.

In this study, we performed a complete analysis of MDR1 coding exon sequences (2-28) in 100 DNA samples obtained from health subjects recruited from different parts of Jordan. Table 1 shows the rare detected variants and their frequencies. Four of the rare variants are synonymous mutations and the remaining are missense mutations. All rare variants were detected in heterozygous genotypes.

Table 1. Frequencies of $M D R 1$ gene variants in the Jordanian population.

\begin{tabular}{lclll}
\hline SNP identification No. & mRNA position & Variation & Function & Allele frequency \\
\hline Rs28364274 & $\underline{4244}$ & $\mathrm{~A}>\mathrm{G}$ & Missense: Ile>Val & 0.005 \\
rs41309228 & $\underline{\underline{3903}}$ & $\mathrm{G}>\mathrm{T}$ & Missense: Ser $>$ Ile & 0.005 \\
This study & $\underline{2332}$ & $\mathrm{~T}>\mathrm{A}$ & Synonymous: Thr & 0.005 \\
rs138926696 & $\underline{1692}$ & $\mathrm{~T}>\mathrm{C}$ & Synonymous: Asp & 0.005 \\
rs2229109 & $\underline{1048}$ & $\mathrm{G}>\mathrm{A}$ & Missense: Ile $>$ Asn & 0.005 \\
rs1128502 & $\underline{652}$ & $\mathrm{~A}>\mathrm{T}$ & Synonymous: Gly & 0.005 \\
rs115493381 & $\mathrm{G}>\mathrm{T}$ & Synonymous: Val & 0.005 \\
\hline
\end{tabular}

Table 2 shows the allele and genotype frequencies of common MDR1 variants in the study sample. The genotype frequencies for rs1128503 (C1236T) were 0.23 (CC), $0.41(\mathrm{CT})$, and 0.36 (TT); for rs2032582 (G2677T) they were $0.38(\mathrm{GG}), 0.45(\mathrm{GT}), 0.13$ (TT), 0.03 (GA), and 0.01 (TA); for rs 1045642 (C3435T) they were 0.17 (CC), 0.5 (CT), and 0.33 (TT). All common MDRl genotypic groups were in Hardy-Weinberg equilibrium $(\mathrm{P}>0.05)$.

Table 2. Genotype and allele frequencies of $M D R 1$ gene SNPs in the Jordanian population.

\begin{tabular}{lccc}
\hline Polymorphism & Genotypes (\%) & Alleles (\%) & Hardy-Weinberg P \\
\hline rs1128503 (C1236T) & CC (23) & C (43.5) & \\
& CT (41) & T (56.5) & \\
rs2032582 (G2677T) & TT (36) & G (62) & \\
& GG (38) & T (36) & \\
GT (45) & A (2) & \\
GA (3) & & \\
& TT (13) & & \\
TA (1) & C (42) & \\
rs1045642 (C3435T) & AA (0) & T (58) & \\
& CT (17) & & 0.79 \\
\hline
\end{tabular}

Only one novel variant was detected in a heterozygous sample. This variant (U3075A) is a silent mutation located in exon 21 (synonymous: Thr; Figure 1). 


\section{A}

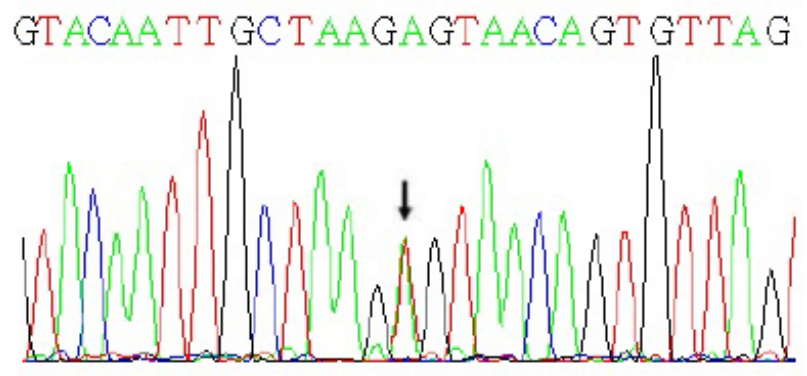

B
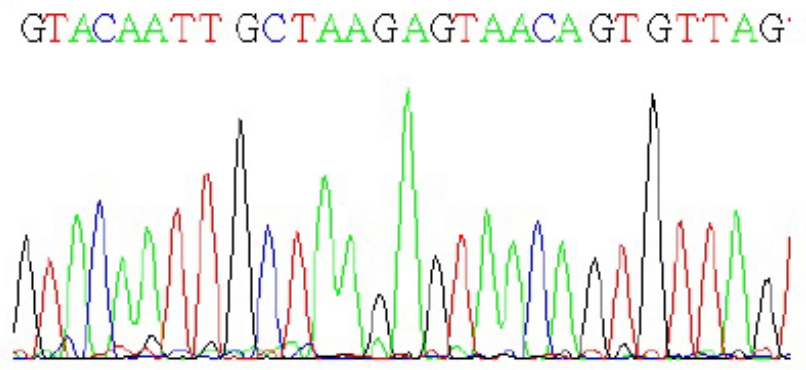

Figure 1. Nucleotide sequence analysis showing T3075A variant in exon 21 of the MDRI gene. The identified novel variant (T3075A) is a synonymous mutation located in exon 21 . The arrow indicates the position of the variant. A. Heterozygous TA genotype. B. Homozygous TT genotype.

\section{DISCUSSION}

The ability to predict a patient's drug response based on their genetic information is emerging as a solution to reduce adverse events and/or improve therapeutic efficacy. Targeting therapies to patients who are most likely to benefit with minimal adverse events will improve patient care and facilitate the approval of new, innovative medicines (Roses, 2008; Katz and Bhathena, 2009). Furthermore, advances in pharmacogenetic working models is associated with superior individualized care for patients suffering from every condition, especially those with cancer (van Schaik, 2008), cardiovascular diseases (D'Andrea et al., 2008), asthma (Hawkins and Peters, 2008), diabetes (Vella and Camilleri, 2008), and psychiatric or neurological disorders (Kadiev et al., 2008). In this study, we scanned the MDR1 coding sequences to identify variations. Three common and 6 rare previously known SNPs were detected. In addition, a novel variant in exon 21 was reported for the first time.

The 3 common SNPs in the Jordanian population were rs1128503, rs2032582, and rs 1045642. These polymorphisms are also common in other human populations but with interethnic differences (Table 3), indicating the ancient origin of these polymorphisms in human history. The $\mathrm{C}$ and $\mathrm{T}$ alleles of rs 1128503 are abundant in the Jordanian population with frequencies of 43.5 and $56.5 \%$. The distribution of this SNP is similar to that reported in the Turkish population (Gumus-Akay et al., 2008). The T allele is significantly less frequent in Serbian, German, and Russian populations (Gaikovitch et al., 2003; Cascorbi, 2011; Milojkovic et al., 
2011) and more frequent in Japanese and Chinese populations (Komoto et al., 2006; Zhang et al., 2008) (see Table 3 for a summary). Similar distributions were also found for the $\mathrm{C}$ and $\mathrm{T}$ alleles of rs 1045642 with frequencies of 42 and $58 \%$. This distribution is similar to that reported in European populations (Gaikovitch et al., 2003; Turgut et al., 2006; Cascorbi, 2011; Milojkovic et al., 2011) but different from that observed in Japanese and Chinese (Komoto et al., 2006; Zhang et al., 2008). The G allele of rs2032582 is enriched in the Jordanian population with 62 vs 36\% for the T allele and $2 \%$ for the A allele. As with rs 1045642, this distribution is similar to that reported in European populations (Gaikovitch et al., 2003; Turgut et al., 2006; Cascorbi, 2011; Milojkovic et al., 2011) but different from that observed in Japanese and Chinese populations (Komoto et al., 2006; Zhang et al., 2008). This could be due to enrichment of the allele A in the Japanese and Chinese ethnicities. Thus, according to the distribution of these common SNPs, the Jordanian population is more closely related to Caucasian than Asian populations.

\begin{tabular}{|c|c|c|c|c|c|c|c|}
\hline \multirow[t]{2}{*}{ Variable } & \multicolumn{2}{|c|}{ C3435T } & \multicolumn{3}{|c|}{ G2677T } & \multicolumn{2}{|c|}{$\mathrm{T} 1236 \mathrm{C}$} \\
\hline & Allele C & Allele $\mathrm{T}$ & Allele G & Allele T & Allele A & Allele C & Allele T \\
\hline Jordan (the current study) & 42 & 58 & 62 & 36 & 2 & 43.5 & 56.5 \\
\hline Serbian (Milojkovic et al., 2011) & 47 & 53 & 53 & 43 & 4 & 54 & 46 \\
\hline German (Cascorbi et al., 2001) & 46 & 54 & 56.5 & 41.5 & 3 & 59.5 & 40.5 \\
\hline Russian (Gaikovitch et al., 2003) & 45.5 & 54.5 & 54.5 & 42 & 3.5 & 52 & 48 \\
\hline Turkish (Turgut et al., 2006; Gumus-Akay et al., 2008) & 46.5 & 53.5 & & & & 47.5 & 52.5 \\
\hline Japanese (Komoto et al., 2006) & 59.5 & 40.5 & 42.5 & 40.5 & 17 & 34.5 & 65.5 \\
\hline Chinese (Zhang et al., 2008) & 56.5 & 43.5 & 42 & 44.5 & 14.5 & 34.5 & 65.5 \\
\hline
\end{tabular}

The most well studied variant of MDR1 is rs 1045642 (C3435T). Although this SNP does not affect the protein sequence of P-glycoprotein, the variant affects expression of the MDR1 gene in the intestine. In addition, studies have indicated that rs1045642 is associated with variable response to chemical therapy. For example, a meta-analysis suggested a correlation between rs 1045642 and tacrolimus pharmacokinetics (Li et al., 2012). Other studies connect this SNP with therapeutic outcomes in gastric, lung, blood, and other cancers ( $\mathrm{Li}$ et al., 2011; Yan et al., 2011) and responses to anti-epileptic (Ponnala et al., 2012) and rheumatoid arthritis drugs (Chen et al., 2011). Moreover, rs1045642 is associated with the risk of breast, renal, blood, and other cancers (Mhaidat et al., 2011; Qian et al., 2012; Wang et al., 2012). Similarly, rs 1128503 is very common and influences imatinib response in patients with chronic myeloid leukemia, tacrolimus response in liver transplant patients ( $\mathrm{Ni}$ et al., 2011; Yu et al., 2011), and risk of ulcerative colitis (Huebner et al., 2009). Finally, the clinical significance of rs 1045642 is evidenced by its association with response to drugs such as cyclosporine and docetaxel (Pan et al., 2009; Wang et al., 2009; Kasuya et al., 2012) and increased colorectal cancer risk (Potocnik et al., 2008). Thus, determination of the frequency of these clinically important SNPs in the MDRl gene is important for prescribing and decreasing the likelihood of adverse reactions and side effects during treatment. In this study, we reported a new MDR1 variant, a silent T3075A substitution. The effect of this variant on the function of MDR1 was not investigated; however, since the mutation is silent, we do not expect a significant effect on the MDR1 protein function. 
Jordan is located in the corner of 3 continents. This geographical location put Jordan on the major trade route between east and west. In addition, the area was exposed to several occupational waves from west and east. This made the Jordanian population open to mixing with other European, African, and Asian populations. Therefore, we expected to find a distribution of MDRI SNPs in the Jordanian population between those observed in other populations. The results of this study support this hypothesis.

This study demonstrated the distribution of $M D R 1$ variants in Jordanians and identified a novel polymorphism. This could form the foundation of future investigations of the relationships between $M D R 1$ polymorphisms and various diseases and drug responses.

\section{ACKNOWLEDGMENTS}

The authors thank Mr. Ma'an Odat and Miss Laila AbuHwaleh for their technical efforts. Research supported by a grant to K.H. Alzoubi from the Scientific Research Funds, Amman, Jordan.

\section{REFERENCES}

Cascorbi I (2011). P-glycoprotein: tissue distribution, substrates, and functional consequences of genetic variations. Handb. Exp. Pharmacol. 201: 261-283.

Chelule PK, Gordon M, Palanee T, Page T, et al. (2003). MDR1 and CYP3A4 polymorphisms among African, Indian, and white populations in KwaZulu-Natal, South Africa. Clin. Pharmacol. Ther. 74: 195-196.

Chen J, Chen L, Mao N and Liu Y (2011). Association of the MDR1 3435 polymorphism in patients with refractory rheumatoid arthritis in a Chinese population. Rheumatol. Int. 32: 3127-3130.

D'Andrea G, D'Ambrosio R and Margaglione M (2008). Oral anticoagulants: Pharmacogenetics Relationship between genetic and non-genetic factors. Blood Rev. 22: 127-140.

Fojo A, Lebo R, Shimizu N, Chin JE, et al. (1986). Localization of multidrug resistance-associated DNA sequences to human chromosome 7. Somat. Cell Mol. Genet. 12: 415-420.

Gaikovitch EA, Cascorbi I, Mrozikiewicz PM, Brockmoller J, et al. (2003). Polymorphisms of drug-metabolizing enzymes CYP2C9, CYP2C19, CYP2D6, CYP1A1, NAT2 and of P-glycoprotein in a Russian population. Eur. J. Clin. Pharmacol. 59: 303-312.

Gumus-Akay G, Rustemoglu A, Karadag A and Sunguroglu A (2008). Genotype and allele frequencies of MDR1 gene C1236T polymorphism in a Turkish population. Genet. Mol. Res. 7: 1193-1199.

Hattori H, Suminoe A, Wada M, Koga Y, et al. (2007). Regulatory polymorphisms of multidrug resistance 1 (MDR1) gene are associated with the development of childhood acute lymphoblastic leukemia. Leuk. Res. 31: 1633-1640.

Hawkins GA and Peters SP (2008). Pharmacogenetics of asthma. Methods Mol. Biol. 448: 359-378.

He T, Mo A, Zhang K and Liu L (2011). ABCB1/MDR1 polymorphism and colorectal cancer risk: a meta-analysis of case-control studies. Colorectal Dis. [Epub ahead of print].

Higgins CF, Callaghan R, Linton KJ, Rosenberg MF, et al. (1997). Structure of the multidrug resistance P-glycoprotein. Semin. Cancer Biol. 8: 135-142.

Huebner C, Browning BL, Petermann I, Han DY, et al. (2009). Genetic analysis of MDR1 and inflammatory bowel disease reveals protective effect of heterozygous variants for ulcerative colitis. Inflamm. Bowel. Dis. 15: 1784-1793.

Kadiev E, Patel V, Rad P, Thankachan L, et al. (2008). Role of pharmacogenetics in variable response to drugs: focus on opioids. Expert. Opin. Drug Metab. Toxicol. 4: 77-91.

Kasuya K, Tsuchida A, Nagakawa Y, Suzuki Y, et al. (2012). Prediction of a side effect and efficacy of adjuvant chemotherapy with gemcitabine for post operative patient of pancreatic cancer by a genetic polymorphism analysis. Hepatogastroenterology 59: 1609-1613.

Katz DA and Bhathena A (2009). Overview of pharmacogenetics. Curr. Protoc. Hum. Genet. Chapter 9: Unit.

Khabour OF, Abdelhalim ES and Abu-Wardeh A (2009). Association between SOD2 T-9C and MTHFR C677T polymorphisms and longevity: a study in Jordanian population. BMC Geriatr. 9: 57.

Kim RB, Leake BF, Choo EF, Dresser GK, et al. (2001). Identification of functionally variant MDR1 alleles among 
European Americans and African Americans. Clin. Pharmacol. Ther. 70: 189-199.

Komoto C, Nakamura T, Sakaeda T, Kroetz DL, et al. (2006). MDR1 haplotype frequencies in Japanese and Caucasian, and in Japanese patients with colorectal cancer and esophageal cancer. Drug Metab. Pharmacokinet. 21: 126-132.

Kuypers DR, de Jonge H, Naesens M and Vanrenterghem Y (2008). Effects of CYP3A5 and MDR1 single nucleotide polymorphisms on drug interactions between tacrolimus and fluconazole in renal allograft recipients. Pharmacogenet. Genomics 18: 861-868.

Lewis DR, Miller ND, Splitt BL, Wu G, et al. (2007). Separating the roles of acropetal and basipetal auxin transport on gravitropism with mutations in two Arabidopsis multidrug resistance-like ABC transporter genes. Plant Cell 19: 1838-1850.

Li Y, Yan PW, Huang XE and Li CG (2011). MDR1 gene C3435T polymorphism is associated with clinical outcomes in gastric cancer patients treated with postoperative adjuvant chemotherapy. Asian Pac. J. Cancer Prev. 12: 2405-2409.

Li Y, Hu X, Cai B, Chen J, et al. (2012). Meta-analysis of the effect of MDR1 C3435 polymorphism on tacrolimus pharmacokinetics in renal transplant recipients. Transpl. Immunol. 27: 12-18.

Mhaidat NM, Alshogran OY, Khabour OF, Alzoubi KH, et al. (2011). Multi-drug resistance 1 genetic polymorphism and prediction of chemotherapy response in Hodgkin's Lymphoma. J. Exp. Clin. Cancer Res. 30: 68.

Milojkovic M, Stojnev S, Jovanovic I, Ljubisavljevic S, et al. (2011). Frequency of the C1236T, G2677T/A and C3435T MDR1 gene polymorphisms in the Serbian population. Pharmacol. Rep. 63: 808-814.

Ni LN, Li JY, Miao KR, Qiao C, et al. (2011). Multidrug resistance gene (MDR1) polymorphisms correlate with imatinib response in chronic myeloid leukemia. Med. Oncol. 28: 265-269.

Pan JH, Han JX, Wu JM, Huang HN, et al. (2009). MDR1 single nucleotide polymorphism G2677T/A and haplotype are correlated with response to docetaxel-cisplatin chemotherapy in patients with non-small-cell lung cancer. Respiration 78: $49-55$.

Ponnala S, Chaudhari JR, Jaleel MA, Bhiladvala D, et al. (2012). Role of MDR1 C3435T and GABRG2 C588T gene polymorphisms in seizure occurrence and MDR1 effect on anti-epileptic drug (phenytoin) absorption. Genet. Test. Mol. Biomarkers 16: 550-557.

Potocnik U, Glavac D and Dean M (2008). Common germline MDR1/ABCB1 functional polymorphisms and haplotypes modify susceptibility to colorectal cancers with high microsatellite instability. Cancer Genet. Cytogenet. 183: 28-34.

Qian X, Cao S, Yang G, Dong J, et al. (2012). Variant genotypes of MDR1 C3435T increase the risk of leukemia: evidence from 10 case-control studies. Leuk. Lymphoma 53: 1183-1187.

Roses AD (2008). Pharmacogenetics in drug discovery and development: a translational perspective. Nat. Rev. Drug Discov. 7: 807-817.

Sipeky C, Csongei V, Jaromi L, Safrany E, et al. (2011). Genetic variability and haplotype profile of MDR1 (ABCB1) in Roma and Hungarian population samples with a review of the literature. Drug Metab. Pharmacokinet. 26: 206-215.

Turgut S, Turgut G and Atalay EO (2006). Genotype and allele frequency of human multidrug resistance (MDR1) gene C3435T polymorphism in Denizli province of Turkey. Mol. Biol. Rep. 33: 295-300.

van Schaik RH (2008). CYP450 pharmacogenetics for personalizing cancer therapy. Drug Resist. Updat. 11: 77-98.

Vella A and Camilleri M (2008). Pharmacogenetics: potential role in the treatment of diabetes and obesity. Expert. Opin. Pharmacother. 9: 1109-1119.

Wang Y, Wang C, Li J, Wang X, et al. (2009). Effect of genetic polymorphisms of CYP3A5 and MDR1 on cyclosporine concentration during the early stage after renal transplantation in Chinese patients co-treated with diltiazem. Eur. $J$. Clin. Pharmacol. 65: 239-247.

Wang Y, Chen Q, Jin S, Deng W, et al. (2012). Up-regulation of P-glycoprotein is involved in the increased paclitaxel resistance in human esophageal cancer radioresistant cells. Scand. J. Gastroenterol. 47: 802-808.

Yan PW, Huang XE, Yan F, Xu L, et al. (2011). Influence of MDR1 gene codon 3435 polymorphisms on outcome of platinum-based chemotherapy for advanced non small cell lung cancer. Asian Pac. J. Cancer Prev. 12: 2291-2294.

Yu X, Xie H, Wei B, Zhang M, et al. (2011). Association of MDR1 gene SNPs and haplotypes with the tacrolimus dose requirements in Han Chinese liver transplant recipients. PLoS One 6: e25933.

Zhang Y, Jiang XH, Hu YQ, Li ZR, et al. (2008). MDR1 genotypes do not influence the absorption of a single oral dose of $600 \mathrm{mg}$ valacyclovir in healthy Chinese Han ethnic males. Br. J. Clin. Pharmacol. 66: 247-254. 